- Sodium hypochlorite is a toxic material and should be handled with care.

- Accidents, while rare, can result in distressing and severe complications for the patient.

- In the event of an accident the patient should be fully informed of the potential sequelae and detailed clinical notes kept.

- This case highlights the clinical features practitioners should look for, enabling them to decide on how best to manage the patient. For those that require hospital admission a treatment protocol is discussed.

- A risk assessment should be made prior to using sodium hypochlorite including careful clinical and radiological examination of the tooth, the type of syringe used and the technique employed.

\title{
Severe tissue damage and neurological deficit following extravasation of sodium hypochlorite solution during routine endodontic treatment
}

\author{
R. Witton ${ }^{1}$ and P. A. Brennan ${ }^{2}$
}

Endodontic therapy is a routinely practised clinical procedure with few reported complications. Sodium hypochlorite is often used as an irrigant during this procedure, but severe complications may occur if this solution extravasates beyond the root apex. We present a case demonstrating some of the severe sequelae that can occur following the misuse of sodium hypochlorite during endodontic treatment of an upper lateral incisor, which also resulted in the previously unreported complication of isolated facial nerve weakness.

\section{CASE REPORT}

A 43-year-old woman with an unremarkable medical history attended Accident and Emergency with severe pain and right facial swelling following endodontic therapy by her general dental practitioner two days earlier. The upper right lateral incisor had a buccal discharging sinus and a diagnosis of chronic apical periodontitis made by the GDP. Endodontic therapy was commenced using rubber dam and the root canal system irrigated with $10 \mathrm{ml}$ of sodium hypochlorite of unknown concentration.

During the second cycle of irrigation the patient experienced severe sudden pain and swelling. Treatment ceased, antibiotics were prescribed and a review appointment arranged. Pain and swelling worsened significantly over the next 24 hours resulting in self-referral to Accident and Emergency.

\footnotetext{
$1^{1 *}$ Senior House Officer in Oral and Maxillofacial Surgery, Queen Alexandra Hospital, Portsmouth, P06 3LY;

${ }^{2}$ Consultant Oral and Maxillofacial Surgeon, Professor of Surgery, Queen Alexandra Hospital, Portsmouth, P06 3LY ${ }^{*}$ Correspondence to: Robert Witton

Email:rvwitton@hotmail.co.uk
}

\section{Refereed Paper}

Received 14.01.05; Accepted 18.02.05

doi: 10.1038/sj.bdj.4812414

๑ British Dental Journal 2005; 198: 749-750
On examination there was a firm right facial swelling, which extended from below the border of the mandible up to the right eye (Fig. 1). There was infra-orbital ecchymosis and altered sensation in the distribution of the right infra-orbital nerve as well as weakness of the buccal branch of the facial nerve. This resulted in some

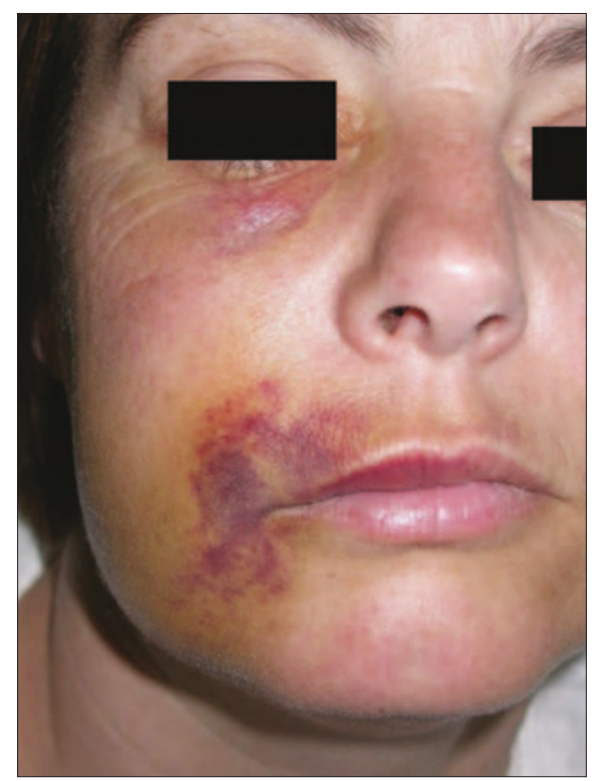

Fig. 1 Initial presentation with swelling and ecchymosis. (Photograph published with the patient's consent) loss of upper lip and cheek function (the corner of the mouth was pulled down by the un-opposed lower lip musculature).

The right upper lip was oedematous with subcutaneous ecchymosis. Opening was limited to $20 \mathrm{~mm}$. Intra-orally there was marked necrosis of the upper right labial mucosa and ulceration of the mucosa of the maxillary alveolus. Radiological examination of the upper right lateral incisor show a previously un-instrumented canal with a large peri-apical radiolucency (Fig. 2).

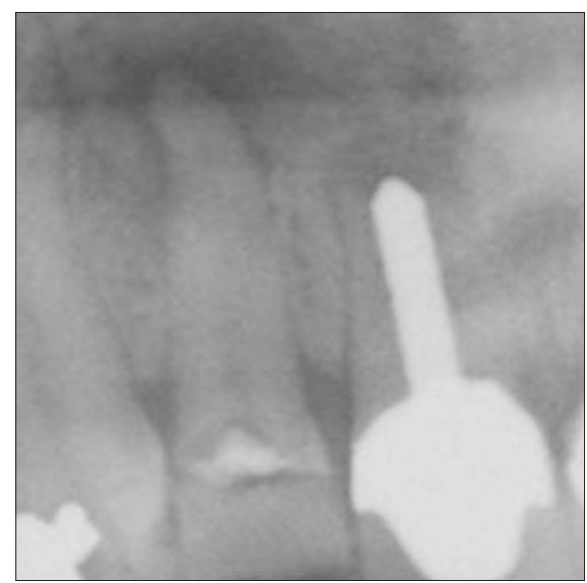

Fig. 2 Radiological examination showing the upper right lateral incisor with periapical radiolucency 
The patient was admitted given intravenous dexamethasone (8 $\mathrm{mg}$ three times a day for two days), and co-amoxiclav (1.2g three times a day) plus regular analgesia for two days. The swelling and pain gradually decreased, and although there was extensive bruising of the skin, no skin necrosis developed. On review one month later the swelling had almost resolved, mouth opening was greatly improved and the patient was pain free (Fig. 3). The infra-orbital nerve paraesthesia was less than on admission, although there was no improvement of her buccal branch facial nerve weakness. No surgical intervention was necessary.

\section{DISCUSSION}

Sodium hypochlorite is a tissue cytotoxic. ${ }^{1,2}$ When it comes into contact with vital tissue, it causes haemolysis, ulceration, inhibits neutrophil migration and damages endothelial and fibroblast cells. ${ }^{1}$

The appearances in this case were consistent with severe soft tissue damage due to a chemical burn, following extrusion of sodium hypochlorite into the periapical tissues. The apical anatomy of this tooth appeared normal so presumably the combination of peri-apical bone destruction due to chronic infection and forced pressure irrigation led to entry of sodium hypochlorite into the soft tissues. It is likely intra-canal microorganisms would also be forced into the tissues posing an infection risk.

Intra-venous steroids were administered in this case due to the rapidly arising painful firm swelling, which continued to increase in size. The profound anti-inflam-

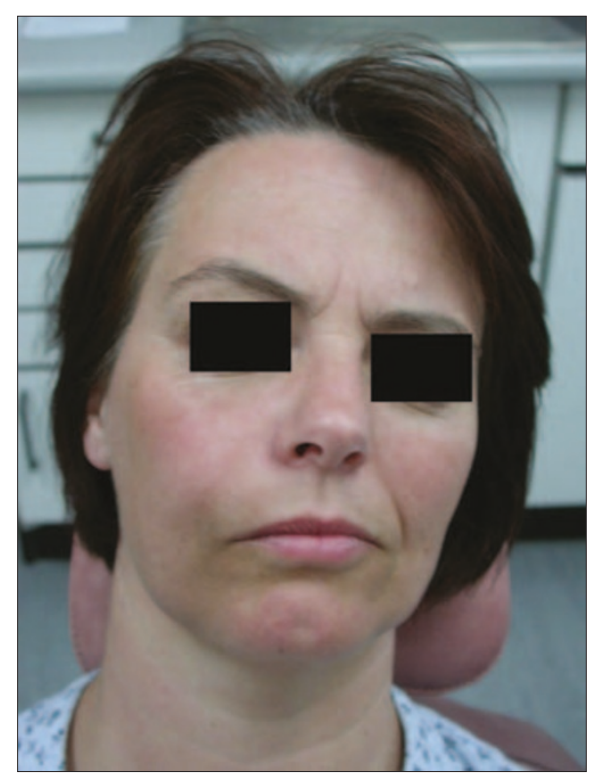

Fig. 3 Appearance of patient at one month. Note the loss of nasolabial angle and down-turning of the right angle of mouth, as a result of buccal branch facial nerve weakness

matory properties of this group of drugs are well documented and have been used in similar case reports. ${ }^{1}$ The use of antibiotics is routinely recommended in these incidents due to the presence of necrotic tissue and the risk of super-infection.

There are only a small number of cases in the literature that have reported postoperative skin complications ${ }^{1-3}$ and altered nerve sensation ${ }^{4}$ arising from the use of sodium hypochlorite in endodontics. However, to our knowledge, this would appear to be the first case of isolated facial nerve weakness following extravasation of sodium hypochlorite solution. It is not possible to comment on whether normal motor function will be regained in this case, although as the infra-orbital nerve paraesthesia was resolving, it is likely that the facial nerve will also recover. Both were likely to be due to hypochlorite damage rather than oedema, since acute dental abscesses with rapidly developing facial swelling are not usually associated with either sensory or motor deficit.

This case serves as a useful reminder to the dental practitioner that sodium hypochlorite solution should be handled with extreme care, and in the unlikely event of an accident, urgent hospital referral should be made. To our knowledge there is no recommended optimal concentration of sodium hypochlorite for endodontic use, with many practioners using a dilute solution of normal household bleach (5.5\% sodium hypochlorite) according to their own preference.

Fortunately this patient did not require any surgical intervention although had the presentation been delayed, the risk of infection and worsening oedema could have been much worse.

1. Gatot A, Arbelle J, Leiberman A, Yanai-Inbar I. Effects of sodium hypochlorite on soft tissues after its inadvertent injection beyond the root apex. J Endod 1991; 17: 573-574.

2. Gernhardt CR, Eppendorf K, Kozlowski A, Brandt M. Toxicity of concentrated sodium hypochlorite used as an endodontic irrigant. Int Endod J 2004; 37: 272-280.

3. Serper A, Ozbek M, Calt S. Accidental sodium hypochlorite-induced skin injury during endodontic treatment. J Endod 2004; 30: 180-181.

4. Gallas-Torreira M M, Reboiras-Lopez M D, GarciaGarcia A, Gandara-Rey J M. Mandibular nerve paresthesia caused by endodontic treatment. Med Oral 2003; 8: 299-303. 